\title{
LAS PERSONAS MAYORES Y LOS PROFESIONALES DE LA ACTIVIDAD FÍSICA Y DEL DEPORTE EN ESPAÑA
}

\author{
Dr. Antonio Campos Izquierdo ${ }^{1}$, Dr. José Emilio Jiménez-Beatty ${ }^{2}$, Dra. María \\ Dolores González Rivera ${ }^{3}$ Dra. María Martín Rodríguez ${ }^{4}$, Dr. David del Hierro \\ Pinés ${ }^{5}$ \\ ${ }^{1}$ Profesor, Universidad Politécnica de Madrid, España, \\ antonio.campos.izquierdo@upm.es \\ ${ }^{2}$ Profesor, Universidad de Alcalá, España,emilio.beatty@uah.es \\ 3rofesora, Universidad de Alcalá, España,marilin.gonzalez@uah.es \\ ${ }^{4}$ Profesora, Universidad Politécnica de Madrid, España,maria.martin@upm.es \\ ${ }^{5}$ Director, Paidesport Center, España, ddelhierro@paidesportcenter.com
}

\section{RESUMEN}

\begin{abstract}
En esta investigación se analiza la existencia de monitores en la actividad fisica y el deporte de las personas mayores de 64 años en España. El objetivo de este estudio es determinar su existencia, en función de las características sociodemográficas de las personas mayores, el tamaño geográfico de los municipios, las actividades practicadas y las organizaciones en las que se realiza la práctica. La metodología utilizada ha consistido en una encuesta seccional aplicada a una muestra de personas mayores en España. Las conclusiones más relevantes que se obtienen son que la presencia de monitores en la actividad fisica y el deporte de las personas mayores es superior (63,8\%), de ahí su importancia, así como que la existencia de monitores es muy superior en mujeres (81,3\%) que en hombres $(37,5 \%)$. Asimismo, se obtiene que según aumenta el tamaño del municipio existe una tendencia a una mayor presencia de monitores. Además, respecto al tipo de actividad se obtiene mucha diversificación y en las entidades deportivas (87,5\%) y en las residencias de mayores (79,5\%) se produce una presencia más grande de monitores.
\end{abstract}

PALABRAS CLAVES: profesionales, ejercicio, monitor, envejecimiento, mayores, actividad física, deporte.

\section{OLDER ADULTS AND SPORT AND PHYSICAL ACTIVITY PROFESSIONALS IN SPAIN}

\section{ABSTRACT}

\begin{abstract}
Presence of monitors in physical activities and sports practiced by adults older than 64 years of age in Spain is analyzed in this research. The objective of this study is to determine the existence of monitors in relation to the sociodemographic features of older adults, the size of municipalities, the activities practiced, and the organizations where they are performed. The methodology used included a cross-sectional survey applied to a sample of older adults in Spain. The most relevant conclusions are that the presence of monitors in physical activities and sports practiced by older adults is dominant $(63.8 \%)$, hence, their importance, and that the presence of monitors is higher for women (81.3\%) than for men (37.5\%). In addition, it is concluded that the bigger the municipality the higher the tendency to have more instructors. Regarding the type of activity, wide diversification is obtained; finally, there is a larger presence of monitors in sports entities (87.5\%) and nursing homes $(79.5 \%)$.
\end{abstract}

KEY WORDS: professionals, exercise, instructor, aging, older adults, physical activity, sport. 


\section{MHSalud}

URL www.una.ac.cr/mhsalud

\section{Introducción}

Durante las últimas décadas, las personas mayores de 64 años han constituido el segmento de población que ha experimentado el crecimiento más acusado en las sociedades occidentales. Esta situación se ha producido especialmente en España, ya que es uno de los países más envejecidos del mundo. Además, en Europa, se prevé un aumento muy considerable del número de estas personas, en las siguientes décadas de este siglo (Martínez, Jiménez-Beatty, Graupera, and Rodríguez, 2006; Jiménez-Beatty, Graupera, Martínez, Campos y Martín, 2007).

Según el Instituto de Mayores y Servicios Sociales (2008) una de las principales características de las personas mayores en España es su disponibilidad de tiempo libre que se traduce en una mayor intensidad y frecuencia de consumo de ocio. En este sentido, Bazo (1996) expresa que debido a los cambios económicos, sociales y culturales acaecidos en las últimas décadas, ellas disponen de más recursos materiales, económicos y sociales, que las generaciones precedentes, para disfrutar del tiempo libre. Dentro de este disfrute, hay que resaltar que según el Instituto Nacional del Estadística (2003) la segunda actividad diaria de ocio más frecuentada por las personas mayores es la realización de ejercicio físico, siendo su dedicación una hora al día.

Asimismo, Jamet (1998) y García-Ferrando (2001) determinan que en la actividad física y el deporte, se está produciendo una gran diversificación y popularidad creciente de estas actividades, entre segmentos cada vez más amplios de la población. Entre esos segmentos de población se encuentran las personas mayores, tradicionalmente marginadas del deporte moderno, pero que, como señalan Mosquera y Puig (2002), cuando ha sido posible (mejora de la calidad de vida, ofertas específicas, etc.) han comenzado a sentirse atraídas por la práctica de estas actividades y lo van incorporando en sus modos de vida. Sin embargo, las personas mayores que practican actividad o ejercicio físico son todavía escasas en comparación a otros grupos sociales debido a la existencia de una serie de circunstancias, obstáculos o barreras.

Por lo que respecta a la práctica de actividad física y deporte de las personas mayores, en España, en el estudio de hábitos deportivos del año 2005 de García-Ferrando (2006), en personas entre 65 y 74 años, se obtuvo que el $17 \%$ son practicantes, y además se observa una evolución con respecto a décadas anteriores.

En el estudio específico sobre personas mayores de 64 años en España de Martínez et ál. (2007a), respecto a sus prácticas de actividad física y deporte semanal, se obtuvo que el $17,4 \%$ practicaban alguna actividad física y/o deportiva semanalmente (demanda establecida), el 12,8\% eran no practicantes pero deseaban realizar al menos alguna actividad semanalmente y sin embargo, por alguna barrera o circunstancia, todavía no podían efectuar dicha actividad (demanda latente) y el $69,8 \%$ eran no practicantes y además no estaban interesados en practicar (demanda ausente) por diferentes barreras o variables inhibidoras.

Al respecto, la mayoría de las investigaciones han detectado como principales barreras, las relacionadas con características personales: el estado de salud y las limitaciones físicas, así como el sentirse mayor (McGuire, 1985; Searle y Iso-Ahola, 1988). A estas investigaciones anteriormente citadas, deben añadirse otras que han planteado la importancia de las características y entornos sociales de las personas mayores como posible fuente de barreras para la práctica (Collins, 2003). Asimismo, otra línea de investigaciones ha encontrado otro 


\section{MHSalud \\ URL www.una.ac.cr/mhsalud}

tipo de barreras relacionadas con deficiencias en la oferta de instalaciones y de actividades deportivas (ausencia, lejanía, características no adecuadas, estructura inadecuada de las clases y su funcionamiento, etc.) para atender sus demandas (Searle y Jackson, 1985; Shephard, 1994; Whaley y Ebbeck, 1997).

Muchas de estas barreras pueden ser reducidas por la adecuada actuación profesional de las personas que dirigen y desarrollan dichas actividades que son profesionales de la actividad física y deporte y con ello conseguir que más personas mayores practiquen actividad física y deporte. Asimismo, los diversos beneficios psicológicos, fisiológicos y sociales que producen esta práctica en gran parte dependen de profesionales de esta área, si éstos no son los adecuados puede existir el riesgo de que se generen problemas con efectos negativos sobre los practicantes mayores (salud, educación, etc.), en lugar de los diversos beneficios directos e indirectos que se producen (Campos, 2007; González, Martín, Jiménez-Beatty, Campos y Del Hierro, 2008).

$\mathrm{Al}$ hacer referencia de las personas profesionales de la actividad física y del deporte, hay que señalar que deben poseer la correspondiente titulación (Campos, 2005) y pertenecer a una de las profesiones establecidas en el anteproyecto de Ley sobre Ordenación del Ejercicio de las Profesiones del Deporte, elaborado por el Consejo Superior de Deportes en el año 2007. Estas son: profesor de educación física, monitor deportivo, entrenador profesional (en un deporte específico) y director deportivo, en este estudio se analiza a las personas monitoras deportivas que trabajan desarrollando las actividad física y deporte en personas mayores de 64 años. En este sentido, Le Roux, Chantelat, and Camy (1999) y el Consejo Superior de Deportes (2000) exponen que el trabajo con personas mayores por parte de estos profesionales es una de las fuentes de empleo en aumento en las próximas décadas.

Asimismo, López (2001), el Consejo Superior de Deportes (2000) y Campos (2009) determinan que además de la formación inicial (titulación de la actividad física y del deporte correspondiente) deben poseer características profesionales: experiencia, formación específica y permanente, así como habilidades sociales y de actitud hacia la actividad, la profesión y el cliente (persona mayor) esto tiene que ver con las características personales de la persona monitora deportivo.

Por su parte, Duda (1991), en una investigación sobre motivaciones de las personas mayores hacia la actividad física y el deporte en referencia a las personas monitoras expresa que deben acomodarse a las circunstancias y características de cada una de las que realizan la actividad y deben estructurar las clases de manera que se sientan realizadas cuando consiguen sus objetivos personales. Además, Hardclastle y Taylor (2001) y Van Norman (1998) sostienen que las personas monitoras deben hacer ver a las personas mayores que a través del ejercicio físico pueden mejorar su calidad de vida y su autoconfianza, así como contribuir a la consecución de objetivos o logros que los motive a continuar.

En este sentido, Shephard (1994) sugiere, entre otras cuestiones, que las personas monitoras en el diseño y desarrollo de la actividad física y deporte deben: partir de los hábitos anteriores de las personas mayores, así como de sus habilidades adquiridas, identificar y resolver barreras tales como la ausencia de compañero o compañera de prácticas, problemas auditivos, cognitivos, emocionales o de conducta y por encima de todo, los ejercicios deben ser personalizados. 


\section{MHSalud \\ URL www.una.ac.cr/mhsalud}

Por lo que respecta a la presencia de personas monitoras, Ispizua y Monteagudo (2002) expresan que la actividad física y el deporte de las personas mayores en grupo suele estar mayoritariamente dirigida y desarrollada por una persona monitora.

Los estudios de Jiménez-Beatty (2002) y de Martínez, Jiménez-Beatty, Graupera y Campos (2007b), establecen que la presencia de personas monitoras en la actividad física y el deporte practicada por las personas mayores es mayoritaria (alrededor del 65\% de las personas), obteniendo también que las mujeres practican principalmente con presencia de personas monitoras (alrededor del 85\%) mientras que en los hombres es escasa (por debajo del 35\%). En relación con la edad, se obtiene que la existencia es superior en todas las edades estudiadas, pero ésta es mayor (por encima del 65\%) en los menores de 75 años. Asimismo, todas las clases sociales, responden que principalmente practican con presencia de una persona monitora, no apreciándose diferencias entre ellas, al igual que se produce respecto a las personas con ingresos suficientes y en las que no lo tienen.

Igualmente, según el modo de organización, en las personas mayores que practican en las entidades deportivas y en los hogares o residencia de mayores, existe una presencia superior de la persona monitora (por encima del 85\%), mientras que existe una actividad autoorganizada en espacios deportivos públicos y abiertos, para toda la población, donde la presencia es muy escasa (menor del 20\%). También, se obtiene que en las actividades acuáticas y el ejercicio físico la presencia de monitor es mayoritaria.

Pues bien, partiendo de este contexto, esta investigación se centra en analizar la presencia de monitores en la actividad física y el deporte practicada por las personas mayores de 64 años en España, teniendo en cuenta que, como se ha explicado anteriormente, es un factor importante para reducir algunas de las barreras en el desarrollo de esta actividad, realizarla adecuadamente y aumentar el número de practicantes. Por ello, el objetivo de este estudio es determinar la existencia de monitores deportivos en la actividad física y el deporte practicada por estas personas mayores, en función de sus características sociodemográficas, el tamaño geográfico de los municipios, las actividades practicadas y las entidades organizadoras.

\section{Metodología}

La metodología ha consistido en una encuesta seccional aplicada a una muestra de personas mayores en España. La encuesta se ha llevado a cabo, mediante la realización de una entrevista personal estandarizada, por medio de cuestionario, en el domicilio de cada una de las personas seleccionadas, de una muestra estadísticamente representativa de las personas de 65 años cumplidos o más, en España (7.484.392 personas, según el Instituto Nacional de Estadística y el Padrón Municipal a 1-1-2006).

\section{Sujetos}

El tamaño de la muestra real es de 933 personas mayores de 65 años cumplidos o más. El intervalo de confianza es de $95,5 \%$, la varianza poblacional es el caso más desfavorable de $\mathrm{p}=50 \%$, luego $\mathrm{q}=50 \%$ y el margen de error permitido de muestreo es de $\pm 3,27 \%$. La afijación de la muestra, ha sido proporcional a la distribución de las personas mayores, según tamaño demográfico de los municipios y según género (Rodríguez, 2000; Sierra, 2001). El tipo de muestreo, ha sido probabilístico de tipo polietápico (Fink, 1995; Miquel, Bigné, Cuenca, Miquel y Lévy, 1997; Bryman, 2004), siendo las unidades de primera etapa los municipios en que residían habitualmente las personas mayores. El resto de unidades de 


\section{MHSalud \\ URL www.una.ac.cr/mhsalud}

etapa han sido en el siguiente orden hasta llegar a las unidades últimas, es decir las personas mayores a entrevistar, las siguientes (dentro de cada municipio seleccionado): barrio, calle, portal, planta, puerta de vivienda. Los 933 sujetos finalmente participantes en el estudio presentaban las siguientes características: según género el $53,1 \%$ son mujeres y el $46,9 \%$ varones; respecto a la edad, el 54,7\% tenían entre 65 y 74 años y el 45,3\% superaban los 75 años; según nivel de estudios, un tercio no tenían ningún tipo de estudios $(36,3 \%)$, la mitad sólo contaba con estudios primarios $(51,5 \%)$, un $6,7 \%$ tenía estudios de bachiller o formación profesional y sólo un 5,4\% habían culminado estudios universitarios; en la distribución según la clase social percibida, la mayoría se consideran de clase media, el $55,3 \%$ de clase media-media y el $27,6 \%$ de media-baja, tan sólo el $8,4 \%$ se consideraba de clase baja y el $8,9 \%$ de clase media-alta o alta; en cuanto al nivel de ingresos la mitad declararon vivir con los ingresos suficientes $(47,4 \%)$, pero la otra mitad vivía con estrecheces y un $5,1 \%$ necesitaba que le ayudaran económicamente.

\section{Instrumentos}

Para captar la información necesaria para los objetivos del estudio, se decidió partir del "Cuestionario de Actividad Física y Personas Mayores" para medir las demandas de actividad física de las personas mayores y otras variables relacionadas, cuyos autores son Graupera, Martínez y Martín (2003), y ya validado en anteriores estudios sobre personas mayores en España (Jiménez-Beatty, Martínez y Graupera, 2006; Jiménez-Beatty, et ál., 2007). Del citado cuestionario fueron seleccionadas las preguntas relacionadas con los objetivos del presente estudio. Con respecto a la validez de contenido fue valorado positivamente por cuatro especialistas externos al equipo de investigación. En el pretest, el instrumento fue administrado a treinta personas pertenecientes al universo estudiado. Todas las personas entendieron las cuestiones y alternativas de respuesta, y no hubo valores perdidos.

La entrevista personal estandarizada por medio de cuestionario se compone de 25 preguntas cerradas de elección múltiple que recogen seis dimensiones relacionadas con: las características sociodemográficas de las personas mayores, el nivel de práctica de actividad física y deporte, las actividades de las personas practicantes en esta área, la demanda de las personas no practicantes pero interesadas en hacerlo, las motivaciones de las personas no practicantes ni interesadas y los conocimientos por parte de las personas mayores sobre los beneficios de la práctica de actividad física y deporte. De este cuestionario, para el desarrollo del estudio, fueron seleccionadas las tres primeras dimensiones citadas, así como los ítems relacionados con los objetivos.

\section{Procedimientos}

La planificación del trabajo de campo fue efectuada durante el mes de mayo de 2006. La selección y formación de las personas entrevistadoras en el cuestionario de la investigación, en las rutas y cuotas a aplicar, en los criterios de selección aleatoria de las personas mayores a entrevistar, tuvo lugar en los meses de octubre y noviembre de 2006. Finalmente, el trabajo de campo se llevó a cabo durante el mes de diciembre de 2006. En él, las personas entrevistadoras aplicaron el cuestionario mediante entrevista personal estructurada cara a cara (Fink, 1995; Bryman, 2004), en el domicilio habitual de la persona mayor seleccionada. Las entrevistas tuvieron una duración promedio de catorce minutos.

\section{Análisis estadístico}

Los análisis de datos han sido efectuados, tras ser tabulados y mecanizados informáticamente. Se ha realizado un análisis descriptivo univariable y bivariable así como 


\section{MHSalud \\ URL www.una.ac.cr/mhsalud}

un análisis inferencial a través de tablas de contingencia que incluyen el valor de Chicuadrado de Pearson y su significación, así como el coeficiente de correlación Phi. Todo ello empleando el paquete de programas SPSS para WINDOWS (V 14.0).

\section{RESULTADOS}

La presencia de monitor en la actividad física y el deporte realizada por personas mayores en España es mayoritaria, ya que éstos están presentes dirigiéndolas y desarrollándolas en el $63,8 \%$ de las personas frente al $36,2 \%$ de las mismas que no tienen esta presencia.

Por su parte, la existencia de monitor en la actividad física y el deporte realizada por mujeres es mayoritaria con el $81,3 \%$, mientras que en las realizadas por los hombres es muy escasa $(37,5 \%)$ (Tabla 1$)$. Se ha obtenido una relación moderada $(\mathrm{Phi}=0,45)$ y significativa (Chicuadrado $\left._{1}=31,805 ; \mathrm{p}=0,00\right)$ en la presencia de monitor respecto al género.

Tabla 1.

Presencia de monitor según género

\begin{tabular}{lcc}
\hline & Presencia de monitor & No presencia de monitor \\
\hline Mujer & $81,3 \%$ & $18,7 \%$ \\
Hombre & $37,5 \%$ & $62,5 \%$ \\
\hline
\end{tabular}

Al analizar la presencia de monitor según la edad de las personas practicantes se obtiene unos datos similares en las dos franjas de edades estudiadas, aunque es levemente mayor la presencia en menores de 75 años (de 65 a 74 años en el 64,1\% y de 75 en adelante en el

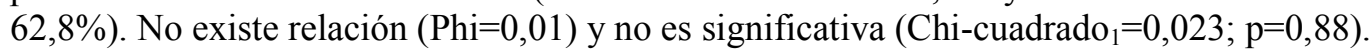

Asimismo, la existencia de monitor según la clase social es muy diversa, ya que se produce en un $65 \%$ de las personas mayores de clase alta/media, en un $57,1 \%$ de personas mayores clase media/media y en un $73,5 \%$ de personas mayores de clase media/baja (tabla 2 ). Esta relación es poco acusada $(\mathrm{Phi}=0,14)$ y no es significativa $($ Chi-cuadrado $2=2,970 ; \mathrm{p}=0,23)$.

Tabla 2.

Presencia de monitor según clase social

\begin{tabular}{lcc}
\hline & Presencia de monitor & No presencia de monitor \\
\hline Clase alta/media & $65 \%$ & $35 \%$ \\
Clase media/media & $57,1 \%$ & $42,9 \%$ \\
Clase media/baja & $73,5 \%$ & $26,5 \%$ \\
\hline
\end{tabular}

En este sentido, respecto al nivel de ingresos la presencia es en un $60,6 \%$ de personas con ingresos suficientes para vivir cómodamente y en un $66,1 \%$ de personas sin ingresos suficientes o con ayudas económicas. No existe relación $(\mathrm{Phi}=0,06)$ y no es significativa (Chi-cuadrado $=0,482 ; \mathrm{p}=0,49$ ).

$\mathrm{Al}$ estudiar la presencia de monitor respecto al tamaño demográfico del municipio se obtiene que según aumenta el tamaño del municipio la presencia es mayor (tabla 3). Igualmente, se obtiene que en municipios menores de 10.000 habitantes la presencia no es mayoritaria (47,6\% de las personas) mientras que a partir de municipios mayores de 10.000 habitantes la 


\section{MHSalud \\ URL www.una.ac.cr/mhsalud}

presencia siempre es mayoritaria y superior al 60\% (Tabla 3). Se ha obtenido una relación poco acusada $(\mathrm{Phi}=0,16)$ y no es significativa $\left(\right.$ Chi-cuadrado $\left.{ }_{3}=3,778 ; \mathrm{p}=0,29\right)$.

Tabla 3.

Presencia de monitor según tamaño demográfico municipal

\begin{tabular}{lcc}
\hline & Presencia de monitor & No presencia de monitor \\
\hline Menor de 10.000 habitantes & $47,6 \%$ & $52,4 \%$ \\
Entre 10.000 y 50.000 habitantes & $60,8 \%$ & $39,2 \%$ \\
Entre 50.001 y 100.000 habitantes & $68,5 \%$ & $31,5 \%$ \\
Mayor de 100.000 habitantes & $70,6 \%$ & $29,4 \%$ \\
\hline
\end{tabular}

La presencia de monitor según la actividad física y el deporte realizada es muy diversificada. En la gimnasia oriental la presencia es en la totalidad de las personas y por encima del $60 \%$ en el ejercicio físico, la actividad física y el deporte con música y en el medio acuático. Por el contrario, las actividades en las que no existe presencia son los juegos de lanzamientos, los desplazamientos urbanos y los deportes y con escasa presencia en la actividad física y el deporte en la naturaleza en el $14,3 \%$ de las personas (tabla 4). Se ha obtenido una relación moderada $(\mathrm{Phi}=0,41)$ y es significativa $($ Chi-cuadrado $7=26,729 ; \mathrm{p}=0,00)$.

Tabla 4.

Presencia de monitor según tipología de actividad fisica y deporte realizado

\begin{tabular}{|c|c|c|}
\hline & Presencia de monitor & No presencia de monitor \\
\hline Ejercicio físico & $64,2 \%$ & $35,8 \%$ \\
\hline Actividad física y deporte con música & $60 \%$ & $40 \%$ \\
\hline Gimnasia oriental & $100 \%$ & $0 \%$ \\
\hline $\begin{array}{l}\text { Actividad física y deporte en el medio } \\
\text { acuático }\end{array}$ & $72,7 \%$ & $27,3 \%$ \\
\hline Juegos de lanzamientos & $0 \%$ & $100 \%$ \\
\hline Desplazamiento urbano & $0 \%$ & $100 \%$ \\
\hline $\begin{array}{l}\text { Actividad física } y \text { deporte en la } \\
\text { naturaleza }\end{array}$ & $14,3 \%$ & $85,7 \%$ \\
\hline Deportes & $0 \%$ & $100 \%$ \\
\hline
\end{tabular}

Respecto al modo de organización, en las entidades deportivas $(87,5 \%$ de las personas mayores) y en los hogares o residencia de mayores $(79,5 \%)$ existe una presencia muy mayoritaria de monitor, mientras que en la actividad autoorganizada la presencia es muy escasa $(4,8 \%)$ (Tabla 5). Se ha obtenido una relación considerable $(\mathrm{Phi}=0,74)$ y es significativa (Chi-cuadrado $3=85,755 ; \mathrm{p}=0,00$ ).

Tabla 5.

Presencia de monitor según modo de organización

\begin{tabular}{lcc}
\hline & Presencia de monitor & No presencia de monitor \\
\hline Entidad deportiva & $87,5 \%$ & $12,5 \%$ \\
Hogar/residencia & $79,5 \%$ & $20,5 \%$ \\
Autoorganización & $4,8 \%$ & $95,2 \%$ \\
\hline
\end{tabular}




\section{MHSalud}

URL www.una.ac.cr/mhsalud

\section{Discusión}

La presencia de monitores en la actividad física y el deporte practicada por las personas mayores de 64 años en España es mayoritaria (63,8\%), cuyo porcentaje es similar a los obtenidos por Jiménez-Beatty (2002) y Martínez, et ál. (2007b). Además, esta tendencia es también descrita por Ispizua y Monteagudo (2002).

Todo esto confirma la importancia expresada por Campos (2009) y Martínez, et ál. (2007a) con respecto a las personas profesionales de la actividad física y del deporte en la práctica con las personas mayores, su influencia de poder reducir las barreras y conseguir así un mayor número de practicantes, así como la necesidad que sean profesionales apropiados para que esto se desarrolle de forma adecuada y sea de calidad, y con ello garantizar los beneficios que pueden generar a estas personas.

La presencia de monitores en las actividades practicadas por las mujeres mayores es superior a la de los hombres, donde dicha tendencia fue también observada por Jiménez-Beatty (2002), Graupera, et ál. (2003) y Martínez, et ál (2007b). Además hay que señalar que Martín, et ál. (2008) determinan que las mujeres mayores presentan más práctica de actividad física y deporte que los hombres.

En relación con la edad, la presencia de los monitores en las actividades practicadas por personas mayores es superior y con porcentajes similares entre las personas mayores de 74 años y las menores de 75 años, al igual que obtiene Jiménez-Beatty (2002).

Respecto a la clase social, la presencia de monitores es mayoritaria en todas la clases sociales, aunque el porcentaje mayor de presencia se produce en la clase media/baja, al contrario que se produce en la práctica de actividad física y deporte, ya que como determina González, et ál. (2008) existe la tendencia en las personas mayores de menor acceso a la práctica a medida que se desciende en la clase social.

$\mathrm{Al}$ analizar la presencia de monitor según el nivel de ingresos, se observa que los ingresos no son una barrera, ya que es mayor en personas sin ingresos suficientes o con ayudas económicas. Aunque se debe tener en cuenta, como demuestran McGuire (1985), Shephard (1994), Martínez, et ál. (2006) y Del Hierro, et ál. (2008) que para la práctica de la actividad física y el deporte el nivel de ingresos puede ser una barrera.

Según aumenta el tamaño demográfico de los municipios existe una mayor presencia de monitor, al igual que obtiene Campos Izquierdo, et ál. (2006).

Respecto a la tipología de la actividad y la presencia de monitor se produce mucha diversificación. Mientras que en la gimnasia oriental la presencia es total, en los juegos de lanzamiento, deportes y el desplazamiento urbano es inexistente. Por su parte, en el ejercicio físico, en la actividad física y el deporte con música y en el medio acuático la presencia es muy mayoritaria; mientras que en la actividad física y el deporte en la naturaleza, esta presencia es escasa. Todo ello tiene la misma tendencia que lo obtenido en los estudios de Jiménez-Beatty (2002) y Campos (2005). 


\section{MHSalud \\ URL www.una.ac.cr/mhsalud}

La presencia de monitores en actividad física y deporte para mayores, desarrolladas por entidades deportivas y hogares o residencias es mayoritaria, al igual que se corrobora con los estudios de Jiménez-Beatty (2002) y Campos (2005).

\section{Conclusiones}

La presencia de monitores en la actividad física y el deporte de las personas mayores de 64 años en España es mayoritaria. Por ello, se debe dar importancia a profesionales de la actividad física y deporte con la finalidad de reducir algunas barreras para la práctica y conseguir un mayor número de personas mayores practicando.

Respecto a características sociodemográficas, la presencia de monitores en la actividad física y deporte de las mujeres mayores es superior a la de los hombres.

Asimismo, hay que resaltar que la presencia de monitores es mayoritaria en todos los niveles de clases sociales y económicas.

Según aumenta el tamaño del municipio existe una tendencia a una mayor presencia de monitor en la actividad física y el deporte de las personas mayores.

La presencia de monitor dependiendo del tipo de actividad realizada es muy diversificada. En la actividad física y el deporte en la naturaleza, en los juegos de lanzamiento, deportes y el desplazamiento urbano es escaso o inexistente. En cambio, en el ejercicio físico, en la actividad física y el deporte con música y en el medio acuático la presencia es muy mayoritaria, llegando a la totalidad en la gimnasia oriental.

Respecto al modo de organización, en las entidades deportivas y los hogares o residencia de mayores se produce una presencia muy superior de monitores.

\section{Referencias bibliográficas}

Bazo, M. T. (1996). Aportaciones de las personas mayores a la sociedad: Análisis sociológico. Revista Española de Investigaciones Sociales, 73, 209-222.

Bryman, A. (2004). Social Research Methods. New York: Oxford University Press.

Campos, A. (2005). Situación profesional de las personas que trabajan en funciones de actividad fisica y deporte en la Comunidad Autónoma Valenciana (2004). Valencia: Universidad de Valencia.

Campos, A. (2007). Los profesionales de la actividad física y del deporte como elemento de garantía y calidad de los servicios. Cultura, Ciencia y Deporte, 7, 51-57.

Campos, A. (2009). Dirección de recursos humanos en las organizaciones de la actividad fisica y del deporte. Madrid: Síntesis.

Collins, M. F. (2003). Social Exclusion from Sport and Leisure. En B. Houlihan (Ed.), Sport and Society (pp. 67-88). Boston: Little Brown.

Consejo Superior de Deportes (2000). El deporte español ante el siglo XXI. Madrid: CSD. 


\section{MHSalud}

URL www.una.ac.cr/mhsalud

Consejo Superior de Deportes (2007). Texto articulado anteproyecto de ley sobre ordenación del ejercicio de determinadas profesiones del deporte. Madrid: CSD.

Del Hierro, D., Campos Izquierdo, A., Jiménez-Beatty, J. E., González, M. D. y Martín, M. (2008). El gasto en actividad física de las personas mayores en España. La demanda establecida. Active Training, 24, 32-33.

Duda, J. (1991). Motivating Older Adults for Physical Activity: It's Never too Late. Journal of Physical Education, Recreation, and Dance, 62, 44-48.

Fink, A. (1995). How to Sample in Surveys. Thousand Oaks: Sage.

García-Ferrando, M. (2001). Los españoles y el deporte: prácticas y comportamientos en la última década del siglo XX. Madrid: CSD.

García-Ferrando, M. (2006). Posmodernidad y Deporte: Entre la individualización y la masificación. Encuesta sobre hábitos deportivos de los españoles 2005. Madrid: CSD.

González, M. D., Martín, M., Jiménez-Beatty, J. E., Campos, A. y Del Hierro, D. (2008). Las necesidades de actividad física de las personas mayores en España. Diferencias según condición social. Habilidad motriz, 31, 16-24.

Graupera, J. L., Martínez, J. y Martín, B. (2003). Factores motivacionales, actitudes y hábitos de práctica de actividad física en las mujeres mayores. Serie ICD de Investigación en Ciencias del Deporte, 35, 181-222.

Hardcastle, S. y Taylor, A. H. (2001). Looking for More than Weight Loss and Fitness Gain: Psychosocial Dimensions among Older Women in a Primary-Care Exercise-Referral Program. Journal of Aging and Physical Activity, 9(3), 313-328.

Instituto de Mayores y Servicios Sociales (2008). Economía y personas mayores. Madrid: IMSERSO.

Instituto Nacional de Estadística (2003). Encuesta de empleo del tiempo 2002-2003. Madrid: I.N.E.

Ispizua, M. y Monteagudo, M. J. (2002). Ocio y deporte en las edades del hombre. En M. García-Ferrando, N. Puig y F. Lagardera (comps.), Sociología del deporte (pp. 231258). Madrid: Alianza Editorial.

Jamet, M. (1998). Changing Patterns of Sporting Practice in France. International Review for the Sociology of Sport, 33(2), 183-187.

Jiménez-Beatty, J. E. (2002). La demanda de servicios de actividad fisica en las personas mayores. Tesis doctoral no publicada. Universidad de León, León.

Jiménez-Beatty, J. E., Martínez, J. y Graupera, J. L. (2006). Las administraciones públicas de la Provincia de Guadalajara y la integración social de las personas mayores a 


\section{MHSalud}

URL www.una.ac.cr/mhsalud

través de los servicios sociales de actividad físico-deportiva. Alcalá de Henares: Universidad de Alcalá-Ibercaja.

Jiménez-Beatty, J. E., Graupera, J. L., Martínez, J., Campos, A., y Martín, M. (2007). Motivational Factors and Physician Advice for Physical Activity in Older Urban Adults. Journal of Aging and Physical Activity, 15, 236-252.

Le Roux, N., Chantelat, P. y Camy, J. (1999). Sport et emploi en Europe. France: Commission Europeenne.

López, A. (2001). La calidad del servicio en instalaciones deportivas acuáticas: un estudio cualitativo. Revista Agua y Gestión, 53, 30-36.

Martín, M., Campos, A., Jiménez-Beatty, J. E. y González, M. D. (2008). Actividades físicas practicadas por las mujeres mayores en España, Kronos, 14, 25-28.

Martínez, J., Jiménez-Beatty, J. E., Graupera, J. L., y Rodríguez, M. L. (2006). Condiciones de vida, socialización y actividad física en la vejez. Revista Internacional de Sociología, 44, 39-62.

Martínez, J., Jiménez-Beatty, J. E., Campos, A., Del Hierro, D., Martín, M. y González, M. D. (2007a). Barreras organizativas y sociales para la práctica de actividad física en la vejez. Motricidad. European Journal of Human Movement, 19, 13-35.

Martínez del Castillo, J., Jiménez-Beatty, J. E., Graupera, J. L. y Campos, A. (2007b). Recursos humanos y actividad física en los actuales practicantes mayores. En P. Montiel, A. Merino, A. Sánchez y A. Heredia (comps.), II Congreso internacional de actividad fisico deportiva para mayores (pp. 450-462). Málaga: I.A.D.

McGuire, F. A. (1985). Constraints in Later Life. En M. G. Wade (Ed.), Constraints on Leisure (pp. 335-353). Springfield: Charles C. Thomas.

Miquel, S., Bigné, E., Lévy, J-P., Cuenca, A.C. y Miquel, M.J. (1997). Investigación de mercados. Madrid: McGraw-Hill.

Mosquera, M. J. y Puig, N. (2002). Género y edad en el deporte. En M. García-Ferrando, N. Puig y F. Lagardera (comps.), Sociología del Deporte (pp. 114-141). Madrid: Alianza Editorial.

Rodríguez, J. (2000). La muestra: teoría y aplicación. En M. García-Ferrando, J. Ibáñez y F. Alvira (eds.), El análisis de la realidad social (pp. 445-483). Madrid: Alianza Editorial.

Searle, M. y Jackson, E. (1985). Socioeconomic Variations in Perceived Barriers to Recreation Participation among Would-be Participants. Leisure Sciences, 7(2): 227249.

Searle, M. y Iso-Ahola, S. (1988). Determinants of Leisure Behaviour among Retired Adults. Therapeutic Recreation Journal, 22(2): 38-46. 


\section{MHSalud}

URL www.una.ac.cr/mhsalud

Shephard, R. (1994). Determinants of Exercise in People Aged 65 Years and Older. En R. Dishman (Ed.), Advances in Exercise Adherence (pp. 344-360). Champaign: Human Kinetics Publishers.

Sierra, R. (2001). Técnicas de investigación social. Teoría y ejercicios. Madrid: Paraninfo.

Van Norman, K. (1998). Motivation and Compliance in Exercise Programs for Older Adults. Journal of Physical Education, Recreation and Dance, 69(8), 24-27.

Whaley, D. E. y Ebbeck, V. (1997). Older Adults' Constraints to Participation in Structured Exercise Classes. Journal of Aging and Physical Activity, 5, 190-212.

Fecha de recepción: 06 de noviembre del 2009.

Fecha de aceptación: 15 de abril del 2010.

Fecha de publicación: 31 de julio de 2010. 